\title{
Frecuencia de leptospirosis y características socio-demográficas en pacientes febriles del norte del Perú
}

\author{
Heber Silva-Díaz, Dunalia N. Llatas-Cancino, Marcos J. Campos-Sánchez, Franklin R. Aguilar-Gamboa, \\ Katya M. Mera-Villasis y Mayra Y. Valderrama-Ayén
}

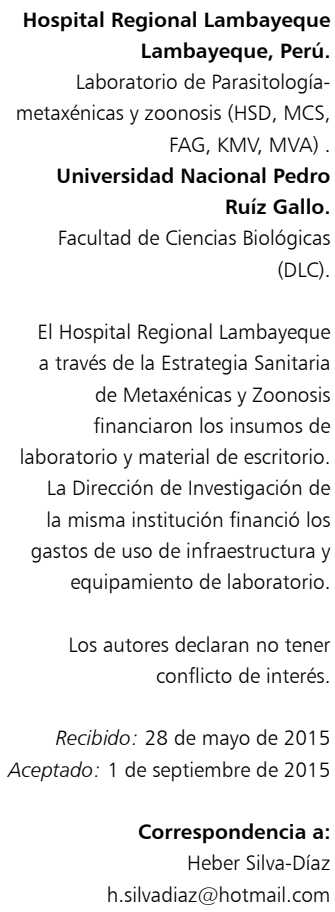

Hospital Regional Lambayeque Lambayeque, Perú. Laboratorio de Parasitologíametaxénicas y zoonosis (HSD, MCS, FAG, KMV, MVA) Universidad Nacional Pedro Ruíz Gallo. Facultad de Ciencias Biológicas (DLC).

El Hospital Regional Lambayeque a través de la Estrategia Sanitaria

de Metaxénicas y Zoonosis financiaron los insumos de laboratorio y material de escritorio. La Dirección de Investigación de la misma institución financió los gastos de uso de infraestructura y equipamiento de laboratorio.

Los autores declaran no tener conflicto de interés.

Recibido: 28 de mayo de 2015 Aceptado: 1 de septiembre de 2015

Correspondencia a: Heber Silva-Díaz h.silvadiaz@hotmail.com

\section{Leptospirosis frequency and socio-demographic characteristics associated in febrile patients from northern Perú}

Indroduction: Leptospirosis is a frequent cause of febrile illness around the world, particularly in tropical and subtropical areas such as Perú. Objective: To estimate the frequency and socio-demographic characteristics associated with leptospirosis in febrile patients in northern Perú. Materials and Methods: A cross sectional study was conducted from January to December 2014 in 143 fever cases admitted to the Hospital Regional Lambayeque, Perú. Structured for socio-demographic information and sanitation questionnaire was used. Serum samples were examined to quantitatively determine the presence of IgM antibodies to leptospira using ELISA IgM. Results: The frequency was $26.6 \%$ (95\% CI 19.3 to 33.8 ) and independently associated features were: be between $20-54$ years (OR: $3.36,95 \%$ CI 1.17 to 9, 64), work in agriculture-livestock (OR: $6.15,95 \%$ CI 1.99 to 19.05), contact with animals (OR: 4,13, IC 95\%: 1,76-9,65) and consumption of unsafe water(OR: 3,33, IC 95\%: 1,49-7,43). Conclusions: This study reveals a high frequency of leptospirosis among febrile patients from northern Perú. These results indicate the need to investigate routinely leptospirosis in febrile patients, especially those exhibiting risk characteristics.

Key words: Leptospirosis, associated features, febrile cases.

Palabras clave: Leptospirosis, características asociadas, casos febriles.

\section{Introducción}

L a leptospirosis es una enfermedad infecciosa bacteriana, producida por varios serovares de Leptospira spp. Es una zoonosis de amplia distribución mundial, común en regiones tropicales y sub-tropicales ${ }^{1-3}$, y es causa frecuente de enfermedad febril en Perú ${ }^{4}$ Los humanos son infectados usualmente con orina de un hospedero infectado, suelo o agua contaminada, o tejido de un animal infectado. Las leptospiras patógenas ingresan al cuerpo a través de las membranas mucosas, conjuntiva, cortes pequeños, abrasiones y posiblemente la piel húmeda, usando diversos mecanismos de patogenicidad poco conocidos aún ${ }^{5}$.

En Perú, la leptospirosis en pacientes febriles se ha comunicado una frecuencia entre 11,1 y $36,6 \% 0^{4,6,7}$, mientras que en población en riesgo entre 1,2 y $64,6 \%{ }^{8-12}$. Asimismo, la leptospirosis es catalogada como hiperendémica en algunas regiones del país, donde la prevalencia de cuadros asintomáticos es alta debido a la frecuente exposición ambiental a esta bacteria ${ }^{9,11-13}$.

En países con áreas tropicales, la población frecuentemente está en riesgo, especialmente donde existe contacto con suelo potencialmente infectado, roedores y otros animales domésticos y clima húmedo. El reservorio más relevante son los roedores ${ }^{1,3,14}$; sin embargo, se incluye como reservorios una amplia variedad de animales domésticos, entre los que destaca el cerdo ${ }^{15}$, caballo ${ }^{16}$, vacuno $^{17-19}$, perro $^{7,13,20}$, oveja ${ }^{21}$, camélidos americanos ${ }^{22} \mathrm{y}$ animales de zoológico ${ }^{23,24}$.

Se considera a la leptospirosis una enfermedad primariamente ocupacional y ha sido asociada con actividades como el trabajo en aguas residuales, ganaderos, agricultores, médicos veterinarios y mineros ${ }^{1,12,25}$. Las condiciones ambientales y demográficas, el estatus socio-económico, la ocupación, el contacto con animales y aguas de recreación son los factores de riesgo más importantes en esta afección ${ }^{12,26,27}$. La identificación de estos factores específicos asociados a la leptospirosis en cada zona geográfica, puede ayudar a incrementar el índice de sospecha diagnóstica de la enfermedad, lo cual cobra importancia debido a la indiferenciación con otras enfermedades febriles.

Por tal motivo, el objetivo de este estudio fue estimar la frecuencia de la leptospirosis y sus características sociodemográficas asociadas, en pacientes febriles admitidos en un hospital regional del Perú. 


\section{Materiales y Métodos}

\section{Diseño y área de estudio}

Se realizó un estudio observacional de corte transversal entre enero y diciembre de 2014 , en pacientes con enfermedad febril (inicio brusco de fiebre, sin foco infeccioso evidente y menos de siete días de evolución), admitidos en el Hospital Regional Lambayeque (HRL), Chiclayo, Perú. El HRL es un hospital de alta complejidad, categoría III-1, que atiende pacientes referidos de la región Lambayeque y regiones aledañas (Amazonas, Cajamarca, San Martín y Piura, principalmente). Estas regiones varían ampliamente en su altitud, desde los $0 \mathrm{msnm}$, en zonas de Lambayeque y Piura, hasta los $3.500 \mathrm{msnm}$ en zonas de Cajamarca. Los pacientes provinieron de áreas urbanas como rurales, con condiciones socio-demográficas y de saneamiento variables.

\section{Población y muestra}

La población fue constituida por pacientes febriles mayores de 6 meses de edad admitidos en el HRL. Los pacientes fueron captados de los servicios de emergencia, consultorio externo, hospitalización y las unidades de cuidados críticos del mencionado hospital durante el año 2014. La muestra incluyó a toda la población: 143 participantes.

\section{Procedimientos}

La recolección de las características socio-demográficas y de saneamiento fue a través de la aplicación de un cuestionario estructurado, aplicado por personal capacitado. Los pacientes mayores de edad respondieron directamente, mientras que en los menores y mayores con incapacidad de hablar, el familiar acompañante o apoderado ayudó a responder las preguntas.

Para certificar el diagnóstico de leptospirosis se obtuvo una muestra de sangre venosa no menor de $1 \mathrm{~mL}$, de la cual se separó el suero, el que fue conservado a $-70^{\circ} \mathrm{C}$ hasta su posterior análisis en el Laboratorio de Inmunología de la Dirección de Investigación del HRL. Las muestras fueron analizadas por la técnica de ELISA, que detectó anticuerpos IgM específicos contra Leptospira interrogans.

Se usó el kit comercial ELISA Leptospira IgM (Virion/Serion, Alemania) para la detección cuantitativa de anticuerpos (Unidades Internacionales por mililitro-UI/ $\mathrm{mL}$ ), el que contó con certificado de calidad para cada lote usado. El fabricante del kit refiere una sensibilidad de $97 \%$ y especificidad de $96 \%$. El protocolo de ensayo siguió las recomendaciones del fabricante y se consideró positivo a leptospirosis títulos de anticuerpos superiores a $20 \mathrm{UI} / \mathrm{mL}$. A los pacientes con menos de 10 días de inicio de síntomas y títulos negativos de anticuerpos, se les solicitó una segunda muestra dos a tres semanas después.

\section{Consideraciones éticas}

El estudio fue revisado y aprobado por el Comité de Ética del HRL. La participación en el estudio fue voluntaria y aceptada a través de la firma de un consentimiento informado. En el caso de los menores de edad, el familiar o apoderado firmó un consentimiento parental. Adicionalmente, los menores de entre 8 y 17 años firmaron un asentimiento. Los participantes que resultaron positivos a leptospirosis fueron derivados al servicio del programa de Metaxénicas y Zoonosis del HRL, con el fin de ser atendidos por un médico infectólogo y recibir un tratamiento apropiado.

\section{Análisis estadístico}

Con los datos obtenidos se estimó la frecuencia de la leptospirosis en la población estudiada. Asimismo, se hizo el análisis descriptivo de las variables socio-demográficas. El análisis de relación entre las características y la leptospirosis se realizó mediante la prueba de Chi cuadrado y la razón de probabilidades (odds ratio, OR); con un intervalo de confianza al $95 \%$ y un valor de "p" significativo menor de 0,05 . Para los cálculos se usaron los programas estadísticos InfoStat/E versión 2008 y GraphPadPrism versión 6.

\section{Resultados}

\section{Frecuencia de leptospirosis y características de la población en estudio}

Participaron en el estudio 143 pacientes febriles admitidos en el HRL. La frecuencia de la leptospirosis mediante la prueba de ELISA IgM fue de 26,6\% (38/143), con un intervalo de confianza al 95\% (IC95\%) de 19,3 a 33,8\%.

La muestra en estudio se caracterizó por tener un predominio masculino (n: $84,58,7 \%$ ) y un segmento etario de entre 20 a 54 años (n: 75; 66,4\%). Asimismo la mayoría de pacientes tenía menos de 6 años de instrucción académica (n: $61 ; 42,7 \%)$ y eran estudiantes o amas de casa. El 61,5\% (n: 88) provenía de la región Lambayeque, seguido por la región Cajamarca con $21,7 \%$ (n: 31$)$ y residían en áreas urbanas el 60,1\% (n: 86). En relación a las condiciones de saneamiento básico, la muestra en estudio se caracterizó por la eliminación de residuos sólidos en depósito municipal (n: 92; 64,3\%), consumo de agua no potable (n: $76 ; 53,2 \%$ ) y eliminación de excretas a través de la red pública (n: 88; 61,5\%) (Tabla 1). La distribución de los casos positivos de leptospirosis según género, grupo etario, procedencia y zona de residencia se muestra en la Figura 1.

\section{Características asociadas}

El análisis bivariado entre las variables socio-demográficas y la leptospirosis (ELISA IgM reactivo), mostró que 
Tabla 1. Características socio-demográficas y de saneamiento de los pacientes febriles admitidos en el Hospital Regional Lambayeque, 2014 (n: 143)

\begin{tabular}{|c|c|c|}
\hline \multicolumn{3}{|l|}{ Características socio-demográficas y de saneamiento } \\
\hline \multicolumn{3}{|l|}{ Género } \\
\hline Femenino & 59 & $(41,3)$ \\
\hline Masculino & 84 & $(58,7)$ \\
\hline \multicolumn{3}{|l|}{ Grupo etario (años) } \\
\hline$\leq 19$ & 43 & $(30,1)$ \\
\hline $20-54$ & 75 & $(52,5)$ \\
\hline$\geq 55$ & 25 & $(17,5)$ \\
\hline \multicolumn{3}{|l|}{ Instrucción (años) } \\
\hline$\leq 6$ & 61 & $(42,7)$ \\
\hline $7-11$ & 56 & $(39,1)$ \\
\hline$\geq 12$ & 26 & $(18,2)$ \\
\hline \multicolumn{3}{|l|}{ Ocupación } \\
\hline Agricultor-Ganadería & 22 & $(15,4)$ \\
\hline Ama de casa & 32 & $(22,4)$ \\
\hline Estudiante & 38 & $(26,5)$ \\
\hline Pre-escolar & 11 & $(7,7)$ \\
\hline Profesional de la salud & 7 & $(4,9)$ \\
\hline Otras ocupaciones & 33 & $(23,1)$ \\
\hline \multicolumn{3}{|l|}{ Región de procedencia } \\
\hline Amazonas & 14 & $(9,8)$ \\
\hline Cajamarca & 31 & $(21,7)$ \\
\hline Lambayeque & 88 & $(61,5)$ \\
\hline Otros & 10 & $(7,0)$ \\
\hline \multicolumn{3}{|l|}{ Zona de residencia } \\
\hline Rural & 57 & $(39,9)$ \\
\hline Urbano & 86 & $(60,1)$ \\
\hline \multicolumn{3}{|l|}{ Eliminación de residuos sólidos } \\
\hline Depósito municipal & 92 & $(64,3)$ \\
\hline Campo abierto & 38 & $(26,6)$ \\
\hline Cuerpos de agua & 13 & $(9,1)$ \\
\hline \multicolumn{3}{|l|}{ Consumo de agua } \\
\hline Potable & 67 & $(46,9)$ \\
\hline No potable & 76 & $(53,2)$ \\
\hline \multicolumn{3}{|l|}{ Eliminación de excretas } \\
\hline Red pública & 88 & $(61,5)$ \\
\hline Campo abierto & 22 & $(15,4)$ \\
\hline Letrina & 33 & $(23,1)$ \\
\hline
\end{tabular}

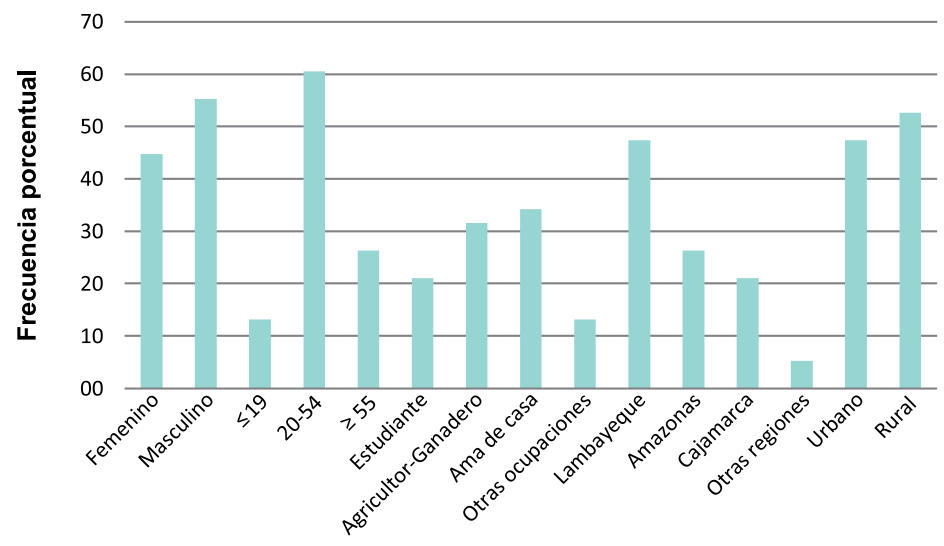

Figura 1. Distribución porcentual de los casos positivos de leptospirosis según género, grupo etario ocupación, procedencia y zona de residencia en pacientes febriles admitidos en el Hospital Regional Lambayeque durante el 2014. tener más de 20 años de edad y ser agricultor/ganadero o ama de casa estuvo relacionado con la mayor frecuencia de leptospirosis. Asimismo, proceder de la región Amazonas también se relacionó con la enfermedad, pero no la región Lambayeque y Cajamarca. No se encontró asociación estadística respecto al género masculino, pocos años de instrucción o la zona de residencia rural (Tabla 2).

Se observó que todas las variables de saneamiento y comportamiento estudiadas estuvieron relacionadas a leptospirosis $(p<0,05)$ : el contacto con agua estancada, agua residual o letrinas y animales. Así también, la eliminación de residuos sólidos en cuerpos de agua, consumo de agua no potable y eliminación de excretas a campo abierto (Tabla 3).

\section{Discusión}

El presente estudio sobre casos de pacientes con enfermedad febril atendidos en el HRL, hospital de alta complejidad al norte de Perú, representa adecuadamente a la población de Lambayeque y de las regiones vecinas, debido a la admisión habitual de pacientes referidos de estas regiones. En estas circunstancias, un estudio hospitalario es el mejor método para captar los casos febriles.

Los reportes sobre la frecuencia de leptospirosis humana en Perú en los últimos 15 años, basados en las pruebas de ELISA IgM y microaglutinación en tubo (MAT), muestran valores de hasta 36,6 y $64,6 \%$, en casos febriles y en población en general, respectivamente ${ }^{7,12}$. No obstante estos valores, las frecuencias varían ampliamente según la región o el tipo de población en que se realizó el estudio ${ }^{4,6,8-11}$.

En el presente estudio, la leptospirosis fue demostrada en 38 pacientes $(26,6 \%)$, evidenciando una alta frecuencia de la enfermedad en las regiones de donde procedieron los pacientes. Nuestros resultados demuestran la naturaleza endémica y la amplia distribución de la leptospirosis en varias regiones del norte del país, las que se suman a otras donde también se ha reportado la enfermedad.

Por otro lado, la frecuencia en este estudio es más alta que las observadas en población hospitalaria y febril de Malasia ${ }^{28}$ e India ${ }^{29}$, los cuales reportaron 8,4 y 4,3\% de leptospirosis, respectivamente. Sin embargo, otro estudio similar en India mostró valores más altos que los encontrados aquí, $52,9 \% \%^{30}$. En África Sub-sahariana la frecuencia de la leptospirosis en pacientes febriles es muy variable, en un rango de $0,0 \%$ en Kenya a $47,5 \%$ en Etiopía ${ }^{26}$. Esto es evidencia de la amplia diversidad y variabilidad de factores que influyen en la presencia de la enfermedad, como la zona demográfica, condiciones climáticas, ambientales y socio-económicas, y múltiples factores de riesgo.

En el presente estudio se pudo observar un ligero 
Tabla 2. Características socio-demográficas asociadas con leptospirosis (ELISA IgM reactivo), en pacientes febriles admitidos en el Hospital Regional Lambayeque, 2014

\begin{tabular}{|c|c|c|c|}
\hline Variable & IgM reactivo/total (\%) & Odds Ratio (IC 95\%) & Valor $\mathbf{p}^{*}$ \\
\hline \multicolumn{4}{|l|}{ Género } \\
\hline Femenino & $17 / 59(28,8)$ & 1,00 & \\
\hline Masculino & $21 / 84(25,0)$ & $0,82(0,39-1,73)$ & 0,611 \\
\hline \multicolumn{4}{|l|}{ Grupo etario (años) } \\
\hline$\leq 19$ & $5 / 43(11,6)$ & 1,00 & \\
\hline $20-54$ & $23 / 75(30,7)$ & $3,36(1,17-9,64)$ & \\
\hline$\geq 55$ & $10 / 25(40,0)$ & $5,07(1,48-17,32)$ & 0,019 \\
\hline \multicolumn{4}{|l|}{ Instrucción (años) } \\
\hline$\geq 12$ & $4 / 26(15,4)$ & 1,00 & \\
\hline $7-11$ & $15 / 56(26,8)$ & $2,01(0,59-6,81)$ & \\
\hline$\leq 6$ & $19 / 61(31,2)$ & $2,49(0,75-8,22)$ & 0,313 \\
\hline \multicolumn{4}{|l|}{ Ocupación } \\
\hline Estudiante & $8 / 49(16,3)$ & 1,00 & \\
\hline Agricultor-Ganadero & $12 / 22(54,6)$ & $6,15(1,99-19,05)$ & \\
\hline Ama de casa & $13 / 32(40,6)$ & $3,51(1,25-9,88)$ & \\
\hline Otras ocupaciones & $5 / 40(12,5)$ & $0,73(0,22-2,44)$ & $<0,001$ \\
\hline \multicolumn{4}{|l|}{ Región de procedencia } \\
\hline Lambayeque & $18 / 88(20,5)$ & 1,00 & \\
\hline Amazonas & 10/14 (71,4) & $9,72(2,73-34,63)$ & \\
\hline Cajamarca & $8 / 31(25,8)$ & $1,35(0,52-3,52)$ & \\
\hline Otros & $2 / 10(20,0)$ & $0,97(0,19-4,98)$ & 0,001 \\
\hline \multicolumn{4}{|l|}{ Zona de residencia } \\
\hline Urbano & $18 / 86(20,9)$ & 1,00 & \\
\hline Rural & $20 / 57(35,1)$ & $2,04(0,97-4,30)$ & 0,061 \\
\hline
\end{tabular}

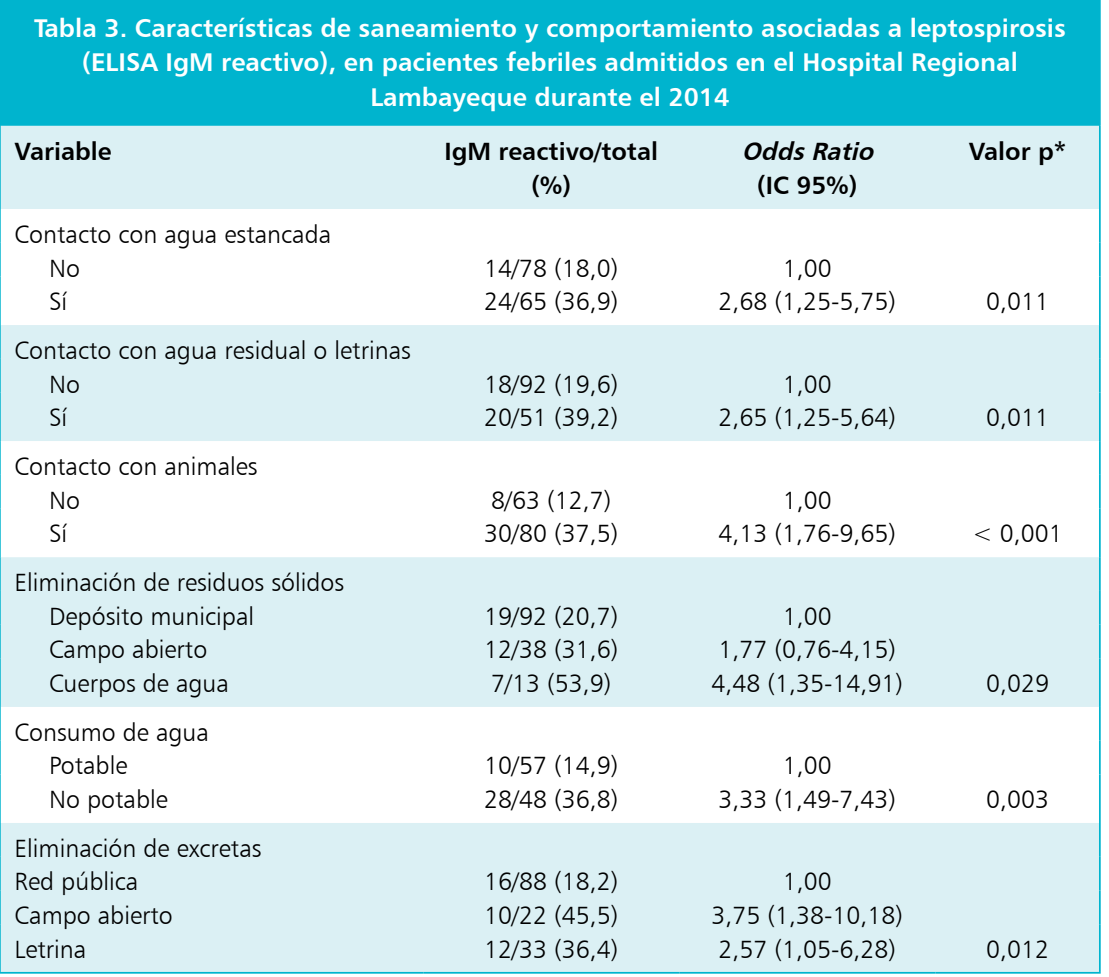

predominio del género masculino en el aporte de casos $(55,3 \%)$, lo cual concuerda con estudios realizados anteriormente en Perú ${ }^{7,12}$ y el mundo ${ }^{28}$, no obstante, esto se debe al mayor número de pacientes varones incluidos en el estudio. Asimismo, la definición de enfermedad ocupacional de la leptospirosis se confirma; por un lado, debido a la mayoritaria frecuencia de casos $(60,5 \%)$ en pacientes con edad laboralmente activa (20-54 años), y por otro, debido a que un importante número de casos se observó en pacientes que se dedican a la agricultura/ ganadería $(31,6 \%)$. Estos hallazgos concuerdan con publicaciones anteriores ${ }^{7,11,12,25}$.

Sin embargo, inesperadamente, las amas de casa aportaron la mayor frecuencia de casos (34\%) en el presente estudio (incluso mayor que los agricultores/ganaderos), hallazgo que ya se ha descrito anteriormente en pacientes febriles en Perú ${ }^{6}$ y en Malasia ${ }^{28}$. No obstante, en estos dos últimos estudios, esta ocupación no fue la más frecuente en el aporte de casos positivos.

En cuanto a los factores asociados, la mayor frecuencia de leptospirosis en pacientes entre 20 y 54 años (OR: 3,36, IC 95\%: 1,17-9,64, p: 0,019), concuerda con estudios anteriores $^{6,11}$. Asimismo, dedicarse a la agricultura/ganadería (OR: 6,15, IC 95\%: 1,99-19,95, p =<0,001) y actividades domésticas (OR: 3,51, IC 95\%: 1,25-9,88, p =<0,001), mostraron también asociación con la enfermedad. Este último hallazgo, puede ser el resultado de varios factores como el contacto con animales domésticos o sus productos (al preparar los alimentos), contacto con agua residual o letrinas, manipulación de residuos sólidos domiciliarios, presencia de roedores en casa, el consumo de agua no potable y otros, los cuales no fueron explorados en el presente estudio.

Por otro lado, la mayor asociación que evidenció la región Amazonas con la enfermedad (OR: 9,72, IC 95\%: 2,73-19,95, p: 0,001), concuerda con estudios realizados en zonas geográficas similares y adyacentes (Jaén y San Martín), donde establecen la alta prevalencia y endemia de la enfermedad. Este dato es importante para las instituciones de salud en el momento de evaluar el diagnóstico clínico de aquellos pacientes febriles que procedan de la mencionada región. Asimismo, las características ambientales asociadas con la enfermedad (contacto con agua estancada, agua residual o letrinas, contacto con animales, y eliminación de excretas y residuos sólidos inadecuadamente), han sido notificados también por otros autores ${ }^{7,11,13}$.

Cabe destacar que, habitar en una zona rural no se asoció con la mayor frecuencia de leptospirosis (OR: 2,04, IC 95\%: 0,97-4,30, $\mathrm{p}=0,061)$, lo que indica que, tanto en la zona rural como urbana, existe la misma probabilidad de adquirir la enfermedad. Esto puede explicarse por las diversas características de riesgo también presentes en las zonas urbanas del área de estudio, como la presencia de 
agua estancada, agua residual anegada, roedores y animales domésticos (perros y gatos). Este hallazgo, aunque lógico al análisis, no ha sido reportado anteriormente en Perú y supone un evidente riesgo de salud pública. Del mismo modo, los pocos años de instrucción no representaron asociación con la enfermedad (OR: 2,49, IC 95\%: $0,75-8,22$, p: 0,313). Esta observación coincide con el encontrado en un estudio anterior ${ }^{12}$.

El presente estudio tuvo algunas limitaciones. Primero, la estimación de la frecuencia fue limitada debido a la falta de confirmación por MAT debido al alto costo, lo cual pudo haber dirigido a una sub o súper estimación de los casos. No obstante, esta limitación fue compensada ampliamente por el uso duplicado de suero, el agudo y el convaleciente (según la historia clínica de la enfermedad); y por la alta sensibilidad (97\%) y especificidad (96\%) del kit usado. En segundo lugar, la modesta cantidad de pacientes enrolados en el estudio (n: 143), lo que limitó algunos cálculos estadísticos y por lo tanto la posibilidad de interpretaciones. Por otro lado, hay aspectos que el estudio no ha podido definir, tanto por el diseño como por el número de muestras. Siendo un estudio transversal no es posible afirmar causalidad en cuanto a las asociaciones encontradas. No obstante, las características identificadas son reconocidas en la trasmisión de la enfermedad y por lo tanto pueden tomarse en cuenta en intervenciones preventivas.

En conclusión, este estudio revela una alta frecuencia de leptospirosis entre los pacientes febriles, indicando la necesidad de investigar la leptospirosis como causa de enfermedad febril, especialmente entre aquellos pacientes que presentan características de riesgo en el norte del Perú. Las características más importantes asociadas a la enfermedad fueron: tener entre 20 a 54 años de edad, dedicarse a la agricultura/ganadería, proceder de la región Amazonas, el contacto con animales y el consumo de agua no potable. La identificación de estas características permitirá proponer intervenciones a fin de reducir el riesgo de infección, además de contribuir al conocimiento actual de la epidemiología de la enfermedad en el Perú.
Agradecimientos. Los autores agradecen a la Estrategia Sanitaria Metaxénicas y Zoonosis del Hospital Regional Lambayeque por el financiamiento y las facilidades para la realización del presente estudio. Asimismo, a las enfermeras y médicos de los servicios del mismo hospital, quienes contribuyeron en la captación y diagnóstico clínico de los pacientes, respectivamente. A la estadístico Génesis Guevara por su opinión profesional al análisis de datos.

\section{Resumen}

Introducción: La leptopsirosis es causa frecuente de enfermedad febril alrededor del mundo, en particular en áreas tropicales y subtropicales como Perú. Objetivo. Estimar la frecuencia de leptospirosis y características socio-demográficas asociadas en pacientes febriles del norte del Perú. Material y Métodos: Se realizó un estudio transversal entre enero y diciembre de 2014 en 143 casos febriles admitidos en el Hospital Regional Lambayeque, Perú. Se usó un cuestionario estructurado para obtener información socio-demográfica y de saneamiento. Se examinaron muestras de suero para determinar cuantitativamente la presencia de anticuerpos IgM contra leptospira usando la técnica de ELISA IgM. Resultados: La frecuencia fue de 26,6\% (IC 95\%: 19,3-33,8) y las características asociadas independientemente fueron: tener entre 20 y 54 años (OR: 3,36, IC 95\%: 1,17-9,64), dedicarse a la agricultura/ganadería (OR: 6,15, IC 95\%: 1,99-19,05), el contacto con animales (OR: 4,13, IC 95\%: 1,76-9,65) y el consumo de agua no potable (OR: 3,33 , IC 95\%: 1,49-7,43). Conclusiones: Este estudio revela una alta frecuencia de leptospirosis entre los pacientes febriles procedentes del norte del Perú. Estos resultados indican la necesidad de investigar la leptospirosis rutinariamente como causa de enfermedad febril, especialmente entre aquellos pacientes que presentan características de riesgo.

\section{Referencias bibliográficas}

1.- Céspedes Z M. Leptospirosis: Enfermedad zoonótica emergente. Rev Peru Med Exp Salud Publica. Instituto Nacional de Salud 2005; 22 (4): 290-307.

2.- Pappas G, Papadimitriou P, Siozopoulou V, Christou L, Akritidis N. The globalization of leptospirosis: worldwide incidence trends. Int J Infect Dis 2008; 12 (4): 351-7.

3.- Dupouey J, Faucher B, Edouard S, Richet H, Kodjo A, Drancourt M, et al. Human leptospirosis: an emerging risk in Europe? Comp Immunol Microbiol Infect Dis 2014; 37
(2): 77-83.

4.- $\quad$ Troyes R L, Fuentes T L, Troyes R M, Canelo D L, García M M, Anaya R E, et al. Etiología del síndrome febril agudo en la provincia de Jaén, Perú 2004-2005. Rev Peru Med Exp Salud Publica. Instituto Nacional de Salud 2006; 23 (1): 5-11.

5.- Adler B. Pathogenesis of leptospirosis: cellular and molecular aspects. Vet Microbiol 2014; 172 (3-4): 353-8.

6.- Vargas-Cuba F, García-Apaico V, Céspedes M, Palomino-Enciso M, Ayala-Huaytalla T. Seroprevalencia y factores asociados con leptospirosis en pacientes con síndrome febril en
Ayacucho, Perú 2005. Rev Peru Med Exp Salud Publica. Instituto Nacional de Salud 2008; 25 (2): 190-4.

7.- Céspedes Z M, Ormaeche M M, Condori P, Balda J L, Glenny A M. Prevalencia de leptospirosis y factores de riesgo en personas con antecedentes de fiebre en la Provincia de Manu, Madre de Dios, Perú. Rev Peru Med Exp Salud Publica. Instituto Nacional de Salud 2003; 20 (4): 80-185.

8.- Platts-Mills J A, La Rochelle P, Campos K, Vinetz J M, Gotuzzo E, Ricaldi J N. Seroprevalencia de leptospirosis en Puente Piedra, Lima en el año 2006. Rev Peru Med Exp 
Salud Publica. Instituto Nacional de Salud 2011; 28 (2): 273-6.

9.- Céspedes Z M, Fernández C R, Rimarachín D R, Taipe S H, Cenepo T J, Mori y Gonzales M, et al. Leptospirosis: Una enfermedad zoonótica hiperendémica en la provincia de Coronel Portillo. Ucayali, Perú. Rev Peru Med Exp Salud Publica. Instituto Nacional de Salud 2004; 21 (2): 62-70.

10.- Ganoza C A, Matthias M A, Saito M, Céspedes M, Gotuzzo E, Vinetz J M. Asymptomatic renal colonization of humans in the peruvian Amazon by Leptospira. PLoS Negl Trop Dis 2010; 4 (2): e612.

11.- Cruz M R, Fernández V F, Arévalo R H. Hiperendemicidad de leptospirosis y factores de riesgo asociados en localidades arroceras del Departamento de San Martín-Perú. Rev Peru Med Exp Salud Publica. Instituto Nacional de Salud 2002; 19 (1): 10-6.

12.- Alarcón-Villaverde J O, Romani-Romani F, Tejada R A, Wong-Chero P, CéspedesZambrano M. Seroprevalencia de leptospirosis y características asociadas en agricultores de arroz de una región tropical del Perú. Rev Peru Med Exp Salud Publica. Instituto Nacional de Salud 2014; 31 (2): 195-203.

13.- Céspedes M, Chu M, Cano E, Huaranca I, Atoche H, Ortiz H, et al. Prevalencia de anticuerpos contra leptospira en personas asintomáticos y en perros de Chancay, Lima 2001. Rev Peru Med Exp Salud Publica. Instituto Nacional de Salud 2007; 24 (4): 343-9.

14.- Muñoz-Zanzi C, Mason M, Encina C, González M, Berg S. Household characteristics associated with rodent presence and Leptospira infection in rural and urban communities from Southern Chile. Am J Trop Med Hyg 2014; 90 (3): 497 506.

15.- Anampa V L, Rivera G H, Falcón P N, Arainga R M, Ramírez V M. Frecuencia de Leptospira spp. en porcinos de crianza tecnificada y de traspatio beneficiados en dos mataderos de Lima. Rev Investig Vet Perú. Universidad
Nacional Mayor de San Marcos 2012; 23 (2): 240-5.

16.- Sotomayor R C, Manchego S A, Chiok C KL, Sandoval C N, Ramírez V M, Rojas M M, et al. Seroprevalencia de anticuerpos contra serovares de Leptospira spp en yeguas de un haras de la ciudad de Lima. Rev Investig Vet del Perú. Universidad Nacional Mayor de San Marcos 2012; 23 (4): 499-503.

17.- Arias C F, Suárez A F, Huanca L W, Rivera G H, Camacho S J, Huanca M T. Prevalencia de leptospirosis bovina en dos localidades de Puno en época de seca y determinación de factores de riesgo. Rev Investig Vet del Perú. Universidad Nacional Mayor de San Marcos 2011; 22 (2): 167-70.

18.- Cachata R S, Suárez A F, Huanca L W, Rivera G H. Prevalencia de anticuerpos contra Leptospira sp. en dos predios de Puno. Rev Investig Vet Perú 2012; 19 (2): 187-91.

19.- Rivera G H, Benito Z A, Ramos C O, Manchego S A. Prevalencia de enfermedades de impacto reproductivo en bovinos de la Estación Experimental de Trópico del Centro de Investigaciones IVITA. Rev Investig Vet Perú 2013; 15 (2): 120-6.

20.- Sacsaquispe C R, Glenny A M, Céspedes Z M. Estudio preliminar de leptospirosis en roedores y canes en salitral, Piura-1999. Rev Peru Med Exp Salud Publica. Instituto Nacional de Salud 2003; 20 (1): 39-40.

21.- Bautista R L, Suárez A F, Huanca L W. Seroprevalencia de leptospirosis en ovinos de dos ganaderías de Puno, Perú. Rev Investig Vet Perú. Universidad Nacional Mayor de San Marcos 2014; 25 (2): 324-8.

22.- Rosadio A R, Véliz A Á, Castillo D H, Yaya L K, Rodríguez H A, Rivera G H, et al. Seroprevalencia a serovares de leptospiras patógenas en alpacas y vicuñas de los departamentos de Huancavelica y Ayacucho, Perú. Rev Investig Vet Perú. Universidad Nacional Mayor de San Marcos 2012; 23 (3): 350-6.

23.- Cueva A E, Rivera G H, Sánchez P N,
Ramírez V M. Incidencia de infección por Leptospira sp. en ronsocos (Hydrochoerus hydrochaeris) en cautiverio en un zoocriadero de Iquitos. Rev Investig Vet Perú. Universidad Nacional Mayor de San Marcos 2010; 21 (1): 106-12.

24.- Montes A D, Rivera G H, Ramírez V M, Ríos M P, Angulo J C, Muñoz D K. Frecuencia de infección por Leptospira sp. en ardillas nuca blanca (Sciurus stramineus) en un zoológico de la ciudad de Lima. Rev Investig Vet Perú. Universidad Nacional Mayor de San Marcos 2011; 22 (1): 67-71.

25.- Dammert B N, Noé M N, Falcón P N, Lopera B L, Rodríguez M P. Exposición a Leptospira sp. en estudiantes de medicina veterinaria al inicio y término de la carrera. Rev Investig Vet Perú. Universidad Nacional Mayor de San Marcos 2009; 20 (1): 114-9.

26.- De Vries S G, Visser B J, Nagel I M, Goris M G A, Hartskeerl R A, Grobusch M P. Leptospirosis in Sub-Saharan Africa: a systematic review. Int J Infect Dis 2014; 28 C: 47-64.

27.- Rafizah A A N, Aziah B D, Azwany Y N, Imran M K, Rusli A M, Nazri S M, et al. Risk factors of leptospirosis among febrile hospital admissions in northeastern Malaysia. Prev Med (Baltim). 2013; 57 Suppl: S11-3.

28.- Rafizah A A N, Aziah B D, Azwany Y N, Imran M K, Rusli A M, Nazri S M, et al. A hospitalbased study on seroprevalence of leptospirosis among febrile cases in north-eastern Malaysia. Int J Infect Dis 2013; 17 (6): e394-7.

29.- Basker P, Kannan P, Kolandaswamy K G. Study on the prevalence of leptospirosis among fever cases reported from private clinics in the urban areas of Villupuram district, Tamil Nadu, India. Osong Public Health Res Perspect 2014; 5 (1): 54-67.

30.- DebMandal M, Mandal S, Pal N K. Serologic evidence of human leptospirosis in and around Kolkata, India: a clinico-epidemiological study. Asian Pac J Trop Med 2011; 4 (12) 1001-6. 